\title{
Determinación del estado hídrico en viña a través de imágenes RGB y multiespectrales adquiridas por un VANT
}

\author{
López García, P. ${ }^{1}$, Ortega Álvarez, J.F. ${ }^{1}$, Moreno Hidalgo, M.A. ${ }^{1}$, Ballesteros González, R. ${ }^{1}$
}

Instituto de Desarrollo Regional, Universidad de Castilla-La Mancha, Albacete, España; Patricia.Lopez@uclm.es; Jose.Ortega@uclm.es; MiguelAngel.Moreno@uclm.es; Rocio.Ballesteros@uclm.es

Resumen: La teledetección se ha convertido en una herramienta muy utilizada en la agricultura de precisión. La existencia de sensores ligeros, calibrados geométrica y radiométricamente, ha hecho que los vehículos aéreos no tripulados (VANTs) sean una de las plataformas más utilizadas en teledetección por obtener datos de muy alta resolución espacial y temporal. En el marco de la viticultura de precisión, haciendo uso de VANTs para la obtención de imágenes aéreas de la cubierta vegetal que proporcionen información agronómicamente útil para hacer un manejo y una gestión del riego sostenible y eficiente frente a la escasez hídrica existente principalmente en zonas áridas y semiáridas, se llevó a cabo un estudio en un viñedo localizado en Fuente-Álamo (Albacete) durante 2018 y 2019. Para obtener un amplio rango de condiciones de estado hídrico de la vid, varias estrategias de riego fueron aplicadas incluyendo regímenes con diferentes niveles de salinidad del agua, estableciendo diferencias en el estado hídrico de la cepa. Los vuelos se hicieron a lo largo del ciclo fenológico usando sensores convencionales (o RGB -red, green, blue-) y multiespectrales a bordo de un VANT, obteniendo ortoimágenes. Estas ortoimágenes fueron segmentadas para incluir solo la vegetación obteniendo el grado de cobertura verde como un parámetro geométrico representativo del desarrollo vegetal del cultivo. El potencial hídrico de tallo fue medido a mediodía con cámaras de presión, y la integral de estrés hídrico fue calculada a partir de estas medidas de estado hídrico, como una variable representativa del efecto acumulado de la intensidad y duración del estrés hídrico desde el comienzo del ciclo de desarrollo hasta el momento de la medida. Modelos de regresión lineal simple usando índices de vegetación basados en la respuesta espectral de la vegetación y el grado de cobertura verde fueron evaluados para predecir la integral de estrés hídrico. Técnicas de regresión no lineal usando redes neuronales artificiales con bandas de los sensores multiespectral y RGB y el grado de cobertura verde como variables predictoras de la integral de estrés hídrico también fueron empleadas. Ambos modelos mostraron que los datos del rango visible (pese a la menor reflectividad de la vegetación en este rango espectral) fueron más útiles para predecir la integral de estrés hídrico que los datos del rango multiespectral, que consideran la reflectividad en el red-edge y en el infrarrojo cercano, proporcionando mejores resultados los modelos no lineales de redes neuronales artificiales con valores de $\mathrm{R}^{2}$ próximos a 1 y errores relativos de $1.5 \%$ o ligeramente superiores. La mayor resolución espacial, calidad radiométrica, facilidad de uso de la cámara RGB, su menor precio y fácil procesamiento de las imágenes RGB hace que estos sensores, utilizados en menor medida con usos agrícolas, sean una buena opción para usarse en la predicción del estado hídrico en viña.

Palabras clave: estrés hídrico; imágenes multiespectrales; imágenes RGB; VANT; viña 


\title{
XXXVIII Congreso Nacional de Riegos CARTAGENA 2021
}

\section{Determination of water status in vineyard using RGB and mul- tispectral images acquired by a UAV}

\author{
López García, P. ${ }^{1}$, Ortega Álvarez, J.F.1, Moreno Hidalgo, M.A. ${ }^{1}$, Ballesteros González, R. ${ }^{1}$
}

Instituto de Desarrollo Regional, Universidad de Castilla-La Mancha, Albacete, Spain; Patricia.Lopez@uclm.es; Jose.Ortega@uclm.es; Migue1Angel.Moreno@uclm.es; Rocio.Ballesteros@uclm.es

\begin{abstract}
Remote sensing has become a widely used tool in precision agriculture. The existence of light sensors, geometrically and radiometrically calibrated, has made unmanned aerial vehicles (UAVs) one of the most used platforms in remote sensing. They obtain very high spatial and temporal resolution data. Within the framework of precision viticulture, the use of UAVs to obtain aerial images of the vineyard provide agronomically useful information to carry out sustainable and efficient irrigation management under a scenario of constant water scarcity mainly in arid and semi-arid areas. In this context, a study was carried out in a vineyard located in Fuente-Álamo (Albacete) during 2018 and 2019. To obtain a wide range of water status conditions of the vine, various irrigation strategies were applied including regimes with different levels of water salinity, establishing differences in the water status of the vine. The flights were made throughout the phenological cycle using conventional (or RGB -red, green, blue-) and multispectral sensors aboard a UAV, obtaining orthoimages. These orthoimages were segmented to include only the vegetation, obtaining the green canopy cover (GCC) as a representative geometric parameter of the plant development. The midday stem water potential was measured with pressure chambers, and the water stress integral was calculated from these measures of water status, as a representative variable of the cumulative effect of the intensity and duration of water stress since the beginning of the development cycle up to the time of measurement. Simple linear regression models using vegetation indices based on the spectral response of the vegetation and the GCC were evaluated to predict the water stress integral. Nonlinear regression techniques using artificial neural networks with bands of the multispectral and RGB sensors and the GCC as predictor variables of the water stress integral were also used. Both models showed that the data from the visible range (despite the lower reflectivity of the vegetation in this spectral range) were more useful to predict the water stress integral than the data from the multispectral range, which considers the reflectivity in the rededge and near infrared, providing better results the non-linear models of artificial neural networks with values of $\mathrm{R}^{2}$ close to 1 and relative errors of $1.5 \%$ or slightly higher. The higher spatial resolution, radiometric quality, ease of use of the RGB camera, its lower price and easy processing of RGB images make these sensors, with less extended use in agriculture, a good option to be used in the prediction of the water status in vineyard.
\end{abstract}

Keywords: multispectral images, RGB images, UAV, vineyard, water stress 


\section{Congreso Nacional de Riegos CARTAGENA 2021}

\section{Introducción}

El estado hídrico del viñedo es el determinante principal tanto en el desarrollo de la cepa como en la composición del vino [1] potencialmente afectados por factores edáficos y ambientales. En zonas áridas y semiáridas, el riego (considerando frecuencia, volumen de agua aplicado y su nivel de sales) juega un papel importante en la determinación del estado hídrico de la cepa [2]. Así, la programación de riego es un factor clave en el equilibrio entre producción y calidad, y en el contexto de escasez de agua, para el manejo sostenible de los recursos hídricos disponibles para usos agrícolas que cada vez son más limitados debido al incremento de la competencia entre los diferentes usos económicos, restricciones ambientales y los efectos del cambio climático [3]. En el marco de la viticultura de precisión, que busca mejorar la eficiencia en la gestión de los recursos disponibles en términos de calidad, producción y sostenibilidad basada en la variabilidad espacial del viñedo dentro de la parcela [4], se emplea el riego deficitario controlado que consiste en reemplazar solo parte de la evapotranspiración potencial del viñedo durante periodos fenológicos previamente establecidos teniendo efectos positivos sobre la calidad de la producción final [5], con una mínima reducción de la producción. La implementación del riego deficitario controlado requiere el control del estado hídrico de la cepa para establecer el momento óptimo de riego. Hay diferentes técnicas para lograr este control, desde métodos que determinan el estado hídrico directamente sobre la planta como es el caso de la conductancia estomática y el potencial hídrico, entre otros, hasta métodos basados en sensores que recogen un gran número de medidas indirectas a lo largo del tiempo, tales como medidas de transpiración, fluctuaciones en el diámetro del tronco o temperatura de la cubierta vegetal o de hoja. Sin embargo, estas medidas son laboriosas, requieren de dispositivos pesados de precio elevado y realizan medidas puntuales pueden no ser representativas de la variabilidad espacial del estado hídrico de la parcela en su conjunto [6].

Hay varias herramientas que son utilizadas por la viticultura de precisión. Una de ellas es el uso de la teledetección, imágenes obtenidas con satélites, aeroplanos, y vehículos aéreos no tripulados (VANTs). Esta información es utilizada para obtener información espacial del cultivo [7]. Los VANTs, con sensores ligeros y de alta calidad geométrica y radiométrica, permiten obtener datos de alta resolución temporal y espacial [8]. Los datos de reflectancia espectral de los sensores a bordo han sido empleados para el control de parámetros biofísicos y bioquímicos, tales como la biomasa, contenido de pigmentos en hoja, el estado hídrico del cultivo, coeficiente de cultivo y la evapotranspiración [9]-[13]. Los datos espectrales son normalmente empleados como una combinación matemática de dos o más bandas para generar índices de vegetación basados en las regiones del visible o RGB (red, green, blue), red-edge e infrarrojo cercano del espectro electromagnético (Pôças et al. 2015). La parte visible del espectro es caracterizada por la baja reflectancia de la cubierta, debido a la fuerte absorción de los pigmentos foliares, principalmente clorofila y carotenoides. La región del infrarrojo cercano es caracterizada por la alta reflectancia y el dominio térmico de la cubierta es caracterizado por su temperatura, de modo que los estomas se cierran bajo una situación de estrés, la transpiración se ralentiza y la temperatura de la hoja aumenta. Por tanto, la temperatura de la hoja o de la cubierta vegetal puede ser utilizada como un indicador de estrés en la planta [16].

Recientes estudios para detectar el estrés hídrico de la viña han usado medidas en el térmico obtenidas de las imágenes aéreas, con una elevada dificultad en la obtención de productos precisos. Otros autores utilizaron índices de vegetación multiespectrales, pero pocos usaron índices de vegetación en el visible, ya que esta región del espectro se caracteriza por la baja reflectancia [14], [17]-[20]. Sin embargo, en este estudio además de índices de vegetación multiespectrales, el incremento de la resolución espacial conduce al uso de índices de vegetación RGB como predictores del estado hídrico de la viña. Esta aproximación permite el uso de sensores de menor coste (cámaras RGB), la obtención de productos 


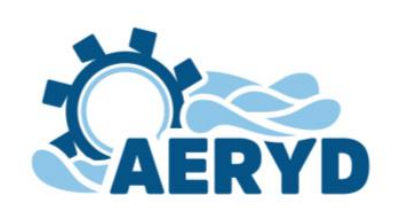

\section{Congreso Nacional de Riegos CARTAGENA 2021}

geomáticos con mayor resolución espacial y un tratamiento fotogramétrico más sencillo comparado con los productos de los sensores multiespectral y térmico.

El grado de cobertura verde (GCV) es un parámetro geométrico que proporciona información sobre el desarrollo vegetativo o el vigor de la cubierta, y es obtenido de las imágenes aéreas. Normalmente se relaciona con el índice de área foliar, la biomasa, la altura de la planta y el volumen de la cubierta vegetal, entre otros parámetros [13], [21]. En este estudio, el GCV será utilizado como predictor del estado hídrico del viñedo junto con la información espectral.

Además de modelos estadísticos de regresión lineal simple y múltiple, el empleo de algoritmos basados en técnicas de inteligencia artificial para tratar conjuntos de datos obtenidos de la teledetección es una buena alternativa debido a su habilidad para aplicar tanto modelos lineales como no lineales [6]. El aprendizaje automático es un tipo de técnica de inteligencia artificial que usa métodos computacionales para aprender directamente del conjunto de datos sin depender de ecuaciones establecidas resolviendo problemas altamente no lineales con la mínima intervención humana [22]. Entre los algoritmos utilizados de aprendizaje automático, las redes neuronales artificiales (RNAs) son ampliamente utilizadas en la simulación de estrés hídrico y otros estudios en agricultura (Poblete et al. 2017, Romero et al. 2018).

Por tanto, el principal objetivo de este estudio es desarrollar modelos predictivos basados en RNAs, que integren información de la cubierta vegetal en el rango del espectro visible y multiespectral de muy alta resolución espacial obtenida con un VANT para evaluar la distribución espacial del estado hídrico del viñedo. Para obtener un amplio rango de condiciones de estado hídrico, se evaluaron varios regímenes de riego, incluyendo regímenes con diferentes niveles de sales, para considerar las diferentes fuentes potenciales de variación en el estado hídrico de la cepa.

\section{Materiales y métodos}

La parcela de viña en la que se llevó a cabo el estudio experimental se encuentra en el término

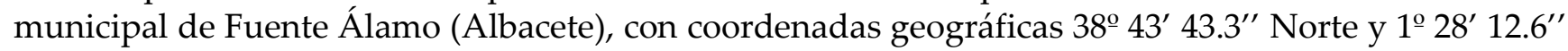
Oeste, con una altitud de $820 \mathrm{~m}$, de 6,5 ha de superficie, ocupando el ensayo 0,6 ha. La parcela se ubica dentro de una zona regable de la Sociedad Agraria de Transformación (SAT) Las Colleras (Figura 1).

A)

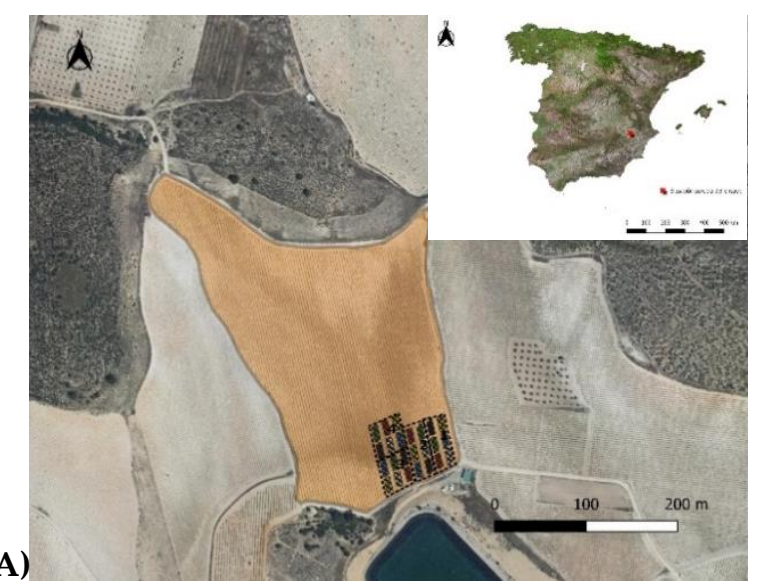

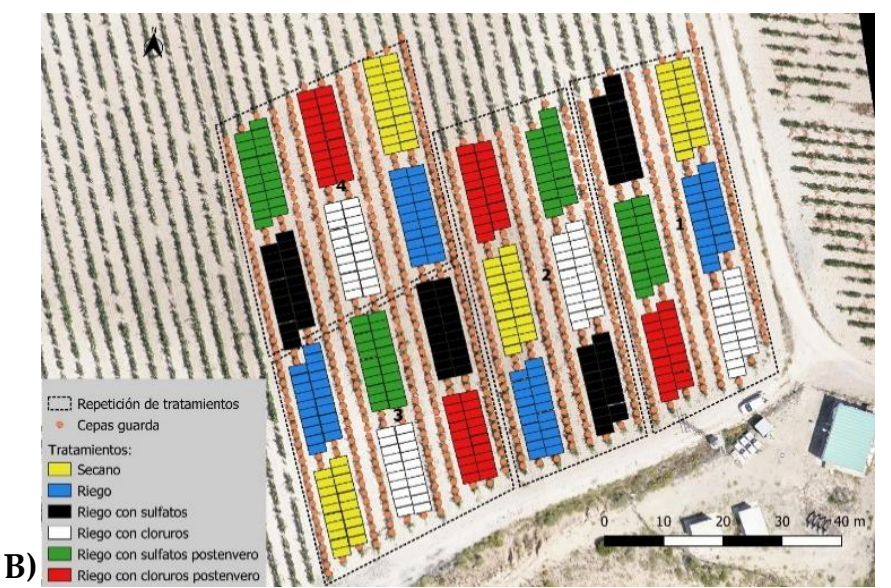

Figura 1. A) Localización del viñedo comercial en Fuente-Álamo (Albacete), y B) distribución de los tratamientos y repeticiones en el diseño experimental. Cada rectángulo coloreado corresponde con una cepa. 


\section{Congreso Nacional de Riegos CARTAGENA 2021}

Se trata de un viñedo de la variedad Monastrell, plantado en 2007, injertado sobre 110-R, con un marco de plantación de $3 \mathrm{~m}$ entre filas y 1,5 m entre cepas dentro de cada fila. El diseño de plantación es en espaldera, orientación Norte-Sur y riego por goteo con goteros autocompensantes de $4 \mathrm{l} / \mathrm{h}$ a $1 \mathrm{~m}$ de distancia. En este viñedo se realizó un ensayo de riego con aguas de diferente calidad (Figura 1 B) durante la campaña de 2018 y 2019. Seis tratamientos (T1-T6) y cuatro repeticiones de cada tratamiento integraron el diseño experimental. Los tratamientos fueron los siguientes: secano (T1), riego con agua de buena calidad (T2), riego con sulfatos añadidos (T3), riego con cloruros añadidos (T4), y T5 y T6 fueron tratamientos de riego con sulfatos y cloruros añadidos, respectivamente, pero iniciando el riego comenzado el envero de la uva. Debido a la escasez hídrica, las restricciones hídricas limitaron el agua disponible para el riego a $1000 \mathrm{~m}^{3} / \mathrm{ha}$, dotación que se aplicó en todos los tratamientos. En la campaña de 2019 el diseño experimental fue simplificado ya que T5 y T6 no se aplicaron porque no se observaron diferencias en cuanto a características agronómicas y de calidad de la uva con respecto a los otros tratamientos de sales añadidas (T3 y T4).

Se midió el potencial hídrico de tallo a mediodía para evaluar el estado hídrico de la viña utilizando una cámara de presión (Model 600, PMS Instrument Company, Albany, OR, EEUU) seis veces en 2018 y siete veces en 2019 en dos hojas de cada tratamiento y repetición. Las medidas de potencial hídrico de tallo se efectuaron los mismos días en los que las imágenes aéreas fueron obtenidas (Tabla 1). El potencial hídrico de tallo caracteriza el estado hídrico de la viña en el momento puntual de la medida, sin embargo, la respuesta espectral de la cubierta vegetal muestra el efecto acumulado de la duración e intensidad del déficit hídrico desde el comienzo del ciclo de desarrollo hasta el momento de la medida. Por tanto, el efecto acumulado del estrés hídrico puede ser representado por la integral de estrés hídrico $\left(S_{\Psi}\right)$, que se calcula como el sumatorio de las medidas del potencial hídrico a lo largo del periodo de estudio [25]. Su cálculo se muestra en la ecuación (1):

$$
S_{\Psi}=\left|\sum_{i=0}^{i=t}\left(\bar{\Psi}_{i, i+1}-c\right) n\right|
$$

Donde: $\bar{\Psi}_{\mathrm{i}, \mathrm{i}+1}$ es la media de dos medidas consecutivas de potencial hídrico; c, es el valor de potencial hídrico menos negativo registrado durante la campaña; y n, es el número de días entre una medida y la siguiente.

Tabla 1. Fechas de medida del potencial hídrico de tallo y de ejecución del vuelo con dron, y estado fenológico del viñedo.

\begin{tabular}{cccc}
\hline Fechas de muestreo & Estado fenológico & Fechas de muestreo & Estado fenológico \\
\hline $15 / 06 / 2018$ & Botones florales (H-I) & $19 / 06 / 2019$ & Comienzo de cuajado (J) \\
$02 / 07 / 2018$ & Cuajado (J) & $04 / 07 / 2019$ & Fin de cuajado (J) \\
$27 / 07 / 2018$ & Racimo cernido (L) & $16 / 07 / 2019$ & Tamaño guisante de la baya (K) \\
$14 / 08 / 2018$ & Comienzo de envero (L-M) & $29 / 07 / 2019$ & Racimo cernido (L) \\
$23 / 08 / 2018$ & Envero (M) & $14 / 08 / 2019$ & Envero (M) \\
$19 / 09 / 2018$ & Maduración (N) & $28 / 08 / 2019$ & Comienzo de maduración (N) \\
& & $18 / 09 / 2019$ & Maduración (N) \\
\hline
\end{tabular}

La imágenes multiespectrales y RGB de alta resolución obtenidas con los sensores SEQUOIA (Parrot, París, Francia) y Sony Ilce-5100 (Sony Corporation, Tokio, Japón) a bordo de un cuadricóptero md4-1000 (Microdrones Inc., Kreuztal, Alemania) se trataron fotogramétricamente para obtener una ortoimagen georreferenciada para toda la parcela con tamaño de pixel de $8 \mathrm{~cm}$ (en el caso de la 


\section{Congreso Nacional de Riegos CARTAGENA 2021}

ortoimagen multiespectral) y $2 \mathrm{~cm}$ (en el caso de la ortoimagen RGB). Estas ortoimágenes se trataron con una versión modificada del programa de visión computacional de cálculo del índice de área foliar [26], obteniendo ortoimágenes con vegetación segmentada (sin suelo y sombras, entre otros) utilizadas para determinar el GCV y varios índices de vegetación (Figura 2).

A)
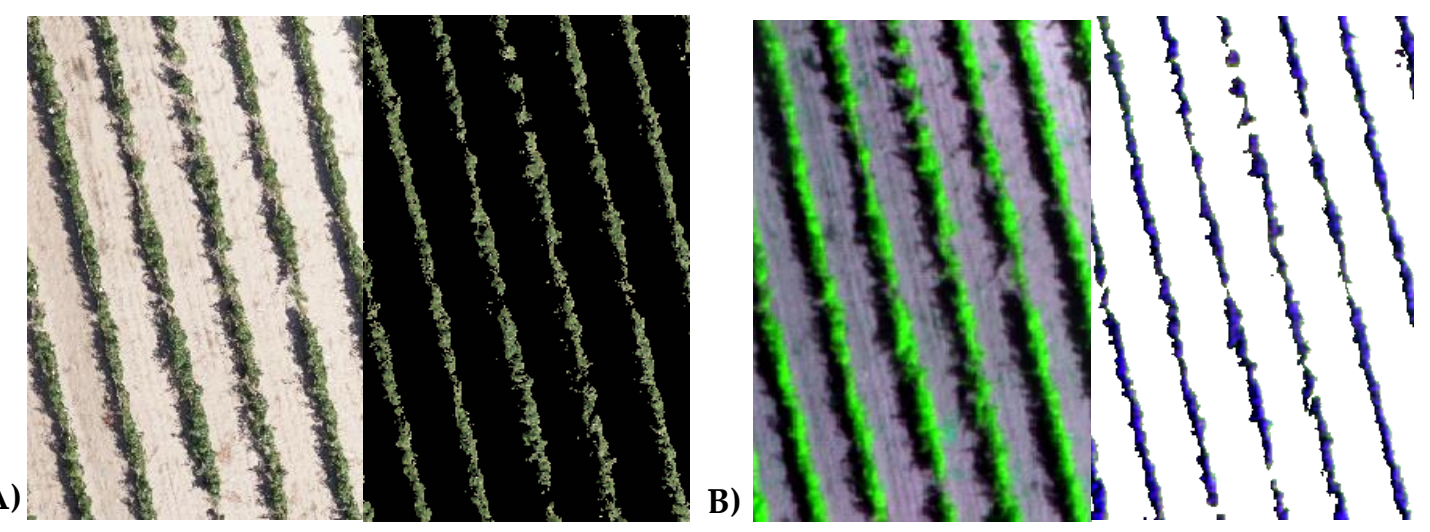

Figura 2. Segmentación de la vegetación en: A) ortoimagen RGB, y B) ortoimagen multiespectral.

El complemento NETLAB del programa MATLAB (Mathworks Inc., Natick, MA, EEUU) se usó para desarrollar y calibrar modelos de RNAs para predecir la $S_{\Psi}$ con distintas combinaciones de bandas. En este estudio, se utilizó una RNA perceptrón multicapa. La estructura de este tipo de RNA está formada por nudos o neuronas interconectadas. Cada conjunto de nudos constituye una capa, y hay tres tipos de capas: a) una capa de entrada, donde cada neurona corresponde a una variable de entrada diferente (en este estudio, el conjunto de bandas separadas de los sensores multiespectrales y RGB y el GCV para cada tratamiento y repetición; b) una capa oculta, donde los datos son procesados; y c) una capa de salida, donde se proporcionan los resultados estimados de la $S_{\Psi}$. Las interconexiones entre neuronas se conocen como pesos sinápticos. Se seleccionó un algoritmo de RNA de retropropagación durante el proceso de calibración para obtener los valores de los pesos sinápticos [22], [27]. Previamente se evaluó el efecto de los tratamientos salinos sobre la reflectancia espectral de la cubierta vegetal y no se observaron diferencias significativas entre ninguna campaña o fechas de muestreo. Por lo tanto, los modelos de RNAs que utilizan las bandas de sensores multiespectrales y RGB para generar la $S_{\Psi}$ se calibraron y validaron para todos los tratamientos juntos. En ambos años, los datos de entrada fueron las tres bandas normalizadas separadas de la cámara RGB para los modelos de RNA RGB y las cuatro bandas separadas para el sensor multiespectral. Se generaron otros dos modelos de RNAs agregando el GCV como dato de entrada. Para cada fecha de muestreo en cada campaña, los datos de entrada totales de las bandas y el GCV junto con los datos de $S_{\Psi}$ medidos se dividieron aleatoriamente en dos grupos con la misma desviación estándar y media para la calibración (60\% del conjunto de datos de entrada total) y validación ( $40 \%$ del conjunto de datos de entrada total). Se implementó un proceso iterativo para lograr este objetivo. La RNA aplicada a los datos de entrada tuvo seis neuronas ocultas y un centenar de iteraciones, que se obtuvieron mediante la metodología desarrollada por [28] para evitar efectos de sobrealimentación del modelo.

Para evaluar la precisión y el funcionamiento de los modelos de RNAs para cada fecha de muestreo en los diferentes años estudiados (2018 y 2019), se emplearon los datos estadísticos sobre el proceso de validación (que utilizó el $40 \%$ de los datos aleatorios), como el coeficiente de determinación $\left(\mathrm{R}^{2}\right)$, el error cuadrático medio (RMSE) y el error relativo (ER), entre los valores de $S_{\Psi}$ medidos y simulados. 


\section{Resultados y discusión}

En las Tablas 2 y 3, para la campaña de 2018 y de 2019, respectivamente, se muestran los resultados de validación de los modelos de RNAs utilizados para predecir la $S_{\Psi}$, empleando como variables predictoras las bandas del espectro visible o multiespectral de los sensores con los que se adquieren las imágenes aéreas de toda la parcela y el GCV. Para 2018, la combinación de bandas independientes RGB actuó mejor como predictora que la combinación de bandas del sensor multiespectral, excepto el 15 de junio. Comparando para una fecha (27/07/2018) los distintos modelos de RNAs aplicados empleando distintos predictores, se observa que utilizando únicamente las bandas independientes del sensor multiespectral se obtiene un valor de $\mathrm{R}^{2}$ de 0.82 que incrementa a 0.97 si además se incluye el GCV multiespectral, no ocurre lo mismo con el ER, que en lugar de disminuir aumenta de $3.27 \%$ a $3.38 \%$, y el RMSE que se mantiene en $0.46 \mathrm{MPa}^{*}$ días, tendencia que se repite para las demás fechas excepto para el 14/08/2018. En cuanto al empleo de las bandas del visible, se obtiene un valor de $\mathrm{R}^{2} 0.98$ que incrementa a 0.99 si además se incluye el GCV, el ER disminuye de $3.06 \%$ a $2.03 \%$, y el RMSE también disminuye de $0.39 \mathrm{MPa}^{*}$ días a $0.27 \mathrm{MPa}^{*}$ días. Tendencia que se repite para las demás fechas. A nivel general, comparando en todas las fechas todos los modelos aplicados, se observa que los modelos que utilizan las bandas RGB y el GCV obtienen mejores parámetros estadísticos (mayores valores de $\mathrm{R}^{2}$ y menores errores). Esto también ocurría en el trabajo previo en el que se analizaron modelos de regresión lineal simple empleando índices de vegetación obtenidos en el rango del espectro multiespectral y visible como predictores [29].

Tabla 2. Resultados de validación ( $R^{2}$ : coeficiente de determinación, RMSE: error medio cuadrático, y ER: error relativo) para los modelos de regresión de redes neuronales artificiales (RNAs) usando bandas RGB y multiespectrales y el grado de cobertura verde $(\mathrm{GCV})$ para simular la integral de estrés hídrico $\left(\mathrm{S}_{\Psi}\right)$ para cada fecha de vuelo en la campaña de 2018. Los valores estadísticos que se muestran en negrita indican el modelo que mejor funciona en cada fecha de muestreo.

\begin{tabular}{|c|c|c|c|c|c|c|c|c|c|c|c|c|c|}
\hline & \multicolumn{3}{|c|}{$\begin{array}{c}\text { RNA con bandas RGB y } \\
\text { GCV }\end{array}$} & \multicolumn{3}{|c|}{ RNA con bandas RGB } & & \multicolumn{3}{|c|}{$\begin{array}{c}\text { RNA con bandas } \\
\text { multiespectrales y GCV } \\
\text { multiespectral }\end{array}$} & \multicolumn{3}{|c|}{$\begin{array}{l}\text { RNA con bandas } \\
\text { multiespectrales }\end{array}$} \\
\hline & $\mathrm{R}^{2}$ & $\begin{array}{c}\text { RMSE } \\
\text { (MPa*días) }\end{array}$ & $\begin{array}{l}\text { ER } \\
(\%)\end{array}$ & $\mathrm{R}^{2}$ & $\begin{array}{c}\text { RMSE } \\
\text { (MPa*días) }\end{array}$ & $\begin{array}{l}\text { ER } \\
(\%)\end{array}$ & & $\mathrm{R}^{2}$ & $\begin{array}{c}\text { RMSE } \\
\text { (MPa*días) }\end{array}$ & $\begin{array}{l}\text { ER } \\
(\%)\end{array}$ & $\mathrm{R}^{2}$ & $\begin{array}{c}\text { RMSE } \\
\text { (MPa*días) }\end{array}$ & $\begin{array}{l}\mathrm{ER} \\
(\%)\end{array}$ \\
\hline $15 / 06 / 2018$ & 0.75 & 0.02 & 4.88 & 0.72 & 0.03 & 6.36 & $15 / 06 / 2018$ & 0.75 & 0.02 & 4.87 & 0.66 & 0.02 & 3.97 \\
\hline 02/07/2018 & 0.85 & 0.24 & 4.75 & 0.84 & 0.28 & 5.66 & $27 / 07 / 2018$ & 0.97 & 0.46 & 3.38 & 0.82 & 0.46 & 3.27 \\
\hline $27 / 07 / 2018$ & 0.99 & 0.27 & 2.03 & 0.98 & 0.39 & 3.06 & $14 / 08 / 2018$ & 0.92 & 1.13 & 4.38 & 0.88 & 1.26 & 5.15 \\
\hline $14 / 08 / 2018$ & 0.99 & 0.58 & 2.48 & 0.94 & 0.88 & 3.65 & $23 / 08 / 2018$ & 0.9 & 1.95 & 6.31 & 0.92 & 1.78 & 6.24 \\
\hline $23 / 08 / 2018$ & 0.99 & 0.51 & 1.56 & 0.99 & 0.72 & 2.17 & $19 / 09 / 2018$ & 0.94 & 2.53 & 5.2 & 0.82 & 2.5 & 5 \\
\hline
\end{tabular}

Para 2019 (Tabla 3), la combinación de bandas independientes RGB actuó mejor como predictora que la combinación de bandas del sensor multiespectral, excepto el 16 y 29 de julio, y el 14 de agosto. Comparando para una fecha (29/07/2019) los distintos modelos de RNAs aplicados empleando distintos predictores se observa que utilizando únicamente las bandas independientes del sensor multiespectral se obtiene un valor de $\mathrm{R}^{2}$ de 0.88 que incrementa a 0.97 si además se incluye el GCV multiespectral, sin embargo, el ER incrementa de $6.39 \%$ a $7.8 \%$, y el RMSE se mantiene en aproximadamente 0.7 $\mathrm{MPa}^{*}$ días, tendencia que no se repite para las demás fechas, pues los errores disminuyen. En cuanto al 


\section{Congreso Nacional de Riegos CARTAGENA 2021}

empleo de las bandas del visible, se obtiene un valor de $\mathrm{R}^{2}$ de 0.96 que disminuye ligeramente a $0.95 \mathrm{si}$ además se incluye el GCV, el ER disminuye de $7.42 \%$ a $7.11 \%$, y el RMSE también disminuye de 0.82 MPa*días a $0.66 \mathrm{MPa}^{*}$ días. Tendencia que se repite para las demás fechas, excepto para el 4 y el 16 de julio. A nivel general, comparando en todas las fechas todos los modelos aplicados, se observa que para cada fecha de muestreo presentan mejores parámetros estadísticos distintos modelos, por ejemplo, para el 16 de julio como mejor modelo predictor sería el que utiliza las bandas multiespectral y el GCV multiespectral, sin embargo, para el 29 de julio el mejor modelo sería el que utiliza las bandas RGB y el GCV. Lo que sí se podría descartar son los modelos que emplean solo las bandas multiespectrales, pues en ninguna fecha de muestreo han presentado el mejor comportamiento. Si bien, estos resultados no permiten generalizar para obtener un modelo predictor, pues en 2018 en todas las fechas de muestreo los modelos que empleaban las bandas RGB y el GCV funcionaron mejor, no ocurriendo lo mismo para 2019, donde los mejores modelos tienen características diferentes, aunque funcionan dentro de parámetros admisibles en los dos años.

Tabla 3. Resultados de validación ( $R^{2}$ : coeficiente de determinación, RMSE: error medio cuadrático, y ER: error relativo) para los modelos de regresión de redes neuronales artificiales (RNAs) usando bandas RGB y multiespectrales y el grado de cobertura verde (GCV) para simular la integral de estrés hídrico $\left(S_{\Psi}\right)$ para cada fecha de vuelo en la campaña de 2019. Los valores estadísticos que se muestran en negrita indican el modelo que mejor funciona en cada fecha de muestreo.

\begin{tabular}{|c|c|c|c|c|c|c|c|c|c|c|c|c|}
\hline & \multicolumn{3}{|c|}{ RNA con bandas RGB y GCV } & \multicolumn{3}{|c|}{ RNA con bandas RGB } & \multicolumn{3}{|c|}{$\begin{array}{c}\text { RNA con bandas } \\
\text { multiespectrales y GCV } \\
\text { multiespectral }\end{array}$} & \multicolumn{3}{|c|}{$\begin{array}{l}\text { RNA con bandas } \\
\text { multiespectrales }\end{array}$} \\
\hline & $\mathrm{R}^{2}$ & $\begin{array}{c}\text { RMSE } \\
\text { (MPa*días) }\end{array}$ & ER (\%) & $\mathrm{R}^{2}$ & $\begin{array}{c}\text { RMSE } \\
\text { (MPa*días) }\end{array}$ & ER (\%) & $\mathrm{R}^{2}$ & $\begin{array}{c}\text { RMSE } \\
\text { (MPa*días) }\end{array}$ & $\begin{array}{l}\mathrm{ER} \\
(\%)\end{array}$ & $\mathrm{R}^{2}$ & $\begin{array}{c}\text { RMSE } \\
\text { (MPa*días) }\end{array}$ & $\begin{array}{l}\text { ER } \\
(\%)\end{array}$ \\
\hline $16 / 07 / 2019$ & 0.98 & 0.39 & 6.45 & 0.93 & 0.39 & 6.15 & 0.97 & 0.16 & 2.34 & 0.99 & 0.28 & 4.6 \\
\hline 29/07/2019 & 0.95 & 0.66 & 7.11 & 0.96 & 0.82 & 7.42 & 0.97 & 0.69 & 7.8 & 0.88 & 0.7 & 6.39 \\
\hline $14 / 08 / 2019$ & 0.98 & 0.65 & 3.76 & 0.97 & 1.1 & 5.6 & 0.99 & 0.29 & 1.57 & 0.98 & 0.74 & 4.14 \\
\hline 28/08/2019 & 0.99 & 0.54 & 2.36 & 0.98 & 0.98 & 3.68 & 0.99 & 0.84 & 3.31 & 0.98 & 1.32 & 4.74 \\
\hline
\end{tabular}

\section{Conclusiones}

El uso de algoritmos de aprendizaje automático, tales como los modelos de RNAs, demostró ser una potente herramienta para el procesamiento de los datos de teledetección obtenidos de VANTs para desarrollar modelos de predicción de $S_{\Psi}$ en los distintos momentos del ciclo de desarrollo del viñedo, ya que los ajustes fueron mejor que aquellos obtenidos de los modelos de regresión lineal simple. Para todas las fechas de muestreo de 2018 y cuatro fechas de 2019, se obtuvieron mejores resultados utilizando bandas RGB como entrada para los modelos de RNAs. El uso de cámaras RGB convencionales aumenta la aplicabilidad de la metodología propuesta debido al menor coste de los sensores RGB, en comparación con los sensores multiespectrales y térmicos, y al procesamiento fotogramétrico más 


\section{Congreso Nacional de Riegos CARTAGENA 2021}

sencillo de las imágenes. Las imágenes RGB de alta resolución permiten la segmentación precisa de la vegetación, lo cual es esencial para evitar los efectos del suelo y obtener valores de GCV precisos.

En este estudio no se generaron modelos de aplicación general, ya que se requeriría una amplia gama de medidas de campo para calibrar un modelo que pudiera aplicarse a una parcela completa. Sin embargo, con unas pocas mediciones de campo, el estado hídrico de una parcela completa se puede estimar con el enfoque propuesto, aunque los mejores modelos en ambos años tengan características diferentes, pues todos funcionan dentro de parámetros admisibles. Los estudios futuros intentarán predecir el estado hídrico en una fecha específica en cualquier año utilizando un modelo calibrado generalizable desarrollado previamente al integrar el número de estaciones analizadas

\section{Referencias}

1. D. I. Jackson and P. B. Lombard, "Environmental and Management Practices Affecting Grape Composition and Wine Quality - A Review," Am. J. Enol. Vitic., vol. 44, no. 4, 1993.

2. J. M. Mirás-Avalos and D. S. Intrigliolo, "Grape Composition under Abiotic Constrains: Water Stress and Salinity," Front. Plant Sci., vol. 8, p. 851, May 2017.

3. J. Hunink et al., "A simplified water accounting procedure to assess climate change impact on water resources for agriculture across different European river basins," Water (Switzerland), vol. 11, no. 10, 2019.

4. A. Matese and S. F. Di Gennaro, "Technology in precision viticulture: A state of the art review," Int. J. Wine Res., vol. 7, no. 1, pp. 69-81, 2015.

5. J. M. Miras-Avalos and E. S. Araujo, "Optimization of vineyard water management: Challenges, strategies, and perspectives," Water (Switzerland), vol. 13, no. 6, pp. 1-32, 2021.

6. S. S. Virnodkar, V. K. Pachghare, V. C. Patil, and S. K. Jha, Remote sensing and machine learning for crop water stress determination in various crops: a critical review, no. 0123456789. Springer US, 2020.

7. M. N. Boukoberine, Z. Zhou, and M. Benbouzid, "A critical review on unmanned aerial vehicles power supply and energy management: Solutions, strategies, and prospects," Appl. Energy, vol. 255, no. August, p. 113823, 2019.

8. L. Pádua et al., "UAS, sensors, and data processing in agroforestry: a review towards practical applications," Int. J. Remote Sens., vol. 38, no. 8-10, pp. 2349-2391, 2017.

9. P. J. Zarco-Tejada et al., "Assessing vineyard condition with hyperspectral indices: Leaf and canopy reflectance simulation in a row-structured discontinuous canopy," Remote Sens. Environ., vol. 99, pp. 271-287, 2005.

10. C. Acevedo-Opazo, B. Tisseyre, H. Ojeda, S. Ortega-Farias, and S. Guillaume, "Is it possible to assess the spatial variability of vine water status?," OENO One, vol. 42, no. 4, p. 203, Dec. 2008.

11. J. Berni, P. J. Zarco-Tejada, L. Suárez, and E. Fereres, "Thermal and narrowband multispectral remote sensing for vegetation monitoring from an unmanned aerial vehicle," IEEE Trans. Geosci. Remote Sens., vol. 47, no. 3, pp. 722-738, Mar. 2009.

12. J. Baluja et al., "Assessment of vineyard water status variability by thermal and multispectral imagery using an unmanned aerial vehicle (UAV)," Irrig. Sci., vol. 30, no. 6, pp. 511-522, Nov. 2012.

13. R. Ballesteros, J. F. Ortega, D. Hernández, and M. Á. Moreno, “Characterization of Vitis vinifera L. canopy using unmanned aerial vehicle-based remote sensing and photogrammetry techniques," Am. J. Enol. Vitic., vol. 66, no. 2, pp. 120-129, May 2015. 


\section{Congreso Nacional de Riegos CARTAGENA 2021}

14. I. Pôças et al., "Predicting grapevine water status based on hyperspectral reflectance vegetation indices," Remote Sens., vol. 7, pp. 16460-16479, 2015.

15. M. Romero, Y. Luo, B. Su, and S. Fuentes, “Vineyard water status estimation using multispectral imagery from an UAV platform and machine learning algorithms for irrigation scheduling management," Comput. Electron. Agric., vol. 147, pp. 109117, Apr. 2018

16. J. M. Costa, O. M. Grant, and M. M. Chaves, “Use of thermal imaging in viticulture: current application and future prospects," in Methodologies and Results in Grapevine Research, S. Delrot, Ed. Springer Science+Business Media B.V., 2010, pp. 136-147.

17. M. Möller et al., “Use of thermal and visible imagery for estimating crop water status of irrigated grapevine,” J. Exp. Bot., vol. 58, no. 4, pp. 827-838, 2007.

18. J. R. Rodríguez-Pérez, D. Riaño, E. Carlisle, S. Ustin, and D. R. Smart, “Evaluation of hyperspectral reflectance indexes to detect grapevine water status in vineyards," Am. J. Enol. Vitic., vol. 58, no. 3, pp. 302-317, Sep. 2007.

19. M. Rossini et al., "Assessing canopy PRI from airborne imagery to map water stress in maize," ISPRS J. Photogramm. Remote Sens., vol. 86, pp. 168-177, 2013.

20. P. J. Zarco-Tejada et al., "A PRI-based water stress index combining structural and chlorophyll effects: Assessment using diurnal narrow-band airborne imagery and the CWSI thermal index," Remote Sens. Environ., vol. 138, pp. 38-50, 2013.

21. R. Ballesteros, J. F. Ortega, D. Hernandez, and M. A. Moreno, “Onion biomass monitoring using UAV-based RGB imaging,” Precis. Agric., vol. 19, no. 5, pp. 840-857, 2018.

22. C. M. Bishop, Pattern recognition and machine learning. New York, USA: Springer, 2006.

23. T. Poblete, S. Ortega-Farías, M. A. Moreno, and M. Bardeen, “Artificial neural network to predict vine water status spatial variability using multispectral information obtained from an unmanned aerial vehicle (UAV)," Sensors, vol. 17, no. 11, 2017.

24. G. Krishna et al., "Comparison of various modelling approaches for water deficit stress monitoring in rice crop through hyperspectral remote sensing," Agric. Water Manag., vol. 213, no. August 2018, pp. 231-244, 2019.

25. I. Buesa, D. Pérez, J. Castel, D. S. Intrigliolo, and J. R. Castel, “Effect of deficit irrigation on vine performance and grape composition of Vitis vinifera L. cv. Muscat of Alexandria," Aust. J. Grape Wine Res., vol. 23, no. 2, pp. 251-259, Jun. 2017.

26. J. I. Córcoles, J. F. Ortega, D. Hernández, and M. A. Moreno, “Estimation of leaf area index in onion (Allium cepa L.) using an unmanned aerial vehicle," Biosyst. Eng., vol. 115, no. 1, pp. 31-42, 2013.

27. M. Kubat, An Introduction to Machine Learning, Second. Springer, 2017.

28. R. Ballesteros, J. F. Ortega, and M. Á. Moreno, “FORETo: New software for reference evapotranspiration forecasting," J. Arid Environ., vol. 124, pp. 128-141, 2016.

29. P. López-García et al., "Assessment of Vineyard Water Status by Multispectral and RGB Imagery Obtained from an Unmanned Aerial Vehicle," Am. J. Enol. Vitic., p. ajev.2021.20063, Jun. 2021. 